\title{
Development of Diffraction Scanning Techniques for Beam Sensitive Polymers.
}

Karen C. Bustillo ${ }^{1}$, Ouliana Panova ${ }^{2}$, Christoph Gammer ${ }^{1,2}$ Edward B. Trigg $^{3}$, X. Chelsea Chen ${ }^{4}$, Lu Yan $^{3}$, Nitash P. Balsara ${ }^{4,5}$, Karen I. Winey ${ }^{3}$, and Andrew M. Minor ${ }^{1,2}$.

${ }^{1 .}$ National Center for Electron Microscopy, Molecular Foundry, and ${ }^{4}$ Materials Sciences Division; Lawrence Berkeley National Laboratory, Berkeley, CA, USA.

2. Department of Materials Science and Engineering and ${ }^{5}$ Department of Chemical and Biomolecular Engineering, University of California, Berkeley, CA, USA.

3. Department of Materials Science and Engineering, University of Pennsylvania, Philadelphia, PA, USA.

We have developed low dose diffraction mapping techniques for application to beam sensitive materials [1]. By exposing the sample with a controlled dose of incident electrons, diffraction from the crystalline regions is captured and used to create contrast in an image reconstructed from the 4-dimensional dataset. These techniques are applied to a model polyethylene sample (PE) as well as a precise acid-containing polyethylene [2] and poly 3-hexylthiophene (P3HT) [3], a conjugated semiconducting polymer used in organic electronic devices. TEM acquisition parameters such as probe size and shape, dose and dose rate, and accelerating voltage are compared with the goal of optimizing the diffraction data acquired. Analysis of the dataset, using segmented virtual apertures of the diffraction spots, allows us to bin the spatial pixels into orientation maps. These techniques are being successfully used across several microscopes including an uncorrected FEI Titan $80-300 \mathrm{kV}$, a Zeiss Libra $80-200 \mathrm{kV}$ with in-line energy filter, and an aberration corrected FEI TEAM I microscope outfitted with a Gatan K2-IS detector.

Beam damage is often the limiting factor in electron microscopy, and this is especially true for many polymers, as well as some 2D materials, low-density frameworks for catalysis, and biological samples. In these cases it is necessary to use imaging techniques that can acquire an in-focus image with the initial low-dose exposure. An additional challenge with polymers that contain predominantly $\mathrm{C}, \mathrm{H}$, and $\mathrm{O}$ is that there is little contrast in bright-field, dark-field, core-loss EELS, or EDS maps. In samples that contain some crystalline phases, diffraction contrast is possible, although in many polymers the diffraction is fleeting, because of damage caused by the electron beam.

By acquiring a short ( 2 to $100 \mathrm{~ms}$ ) convergent beam diffraction pattern at each step of a scanning beam (STEM) image, one acquires a 4-dimensional dataset - each pixel (x,y) has an associated diffraction pattern $\left(x^{\prime}, y^{\prime}\right)$. The diffraction pattern is largely insensitive to focus of the objective lens allowing one to move to an unexposed area and acquire without focusing or pre-exposing the sample. A typical beam convergence angle is $0.5-1 \mathrm{mrad}$, which produces well-separated diffraction disks. Although typical doses (electrons $/ \mathrm{A}^{2}$ ) are high in a converged beam because the probe size is small compared with the exposed area of a conventional parallel TEM beam, the diffraction pattern is efficient at using the incident electrons, and consequently the exposure times can be short and still produce diffraction patterns that can be detected by computer algorithms. Additionally one can adjust the sample height, which creates a variable probe size $(2-30+\mathrm{nm})$, and this facilitates additional dose reduction. The spatial resolution of the reconstructed real space image is determined by the step size of the convergent beam. Post processing of the dataset involves using virtual apertures to select diffraction conditions that build dark field images, which can be combined into an orientation map. 
This technique holds the possibility of allowing us to visualize the size and orientation of the polymer crystallites with a nanoscale probe. For example, we have investigated a high-density polyethylene grown by solution methods and known to have an orthorhombic structure. By indexing the diffraction patterns we find that the polymer lamellae are lying flat with the [001] axis parallel to the electron beam. The stack of diffraction frames includes three possible motifs: 1. Diffraction from a single crystal (Figures 1c and 1e); 2. Diffraction from two or more crystal orientations, which may arise because of stacking that is twisted or because the probe has sampled adjacent crystallites (Figure 1b); 3 . No diffraction, arising from regions that are either amorphous or not oriented (Figure 1a). Figures 1c/1d and 1e/1f shows two representative diffraction patterns paired with the real space image showing where these crystallites are found. From these maps we learn that the crystallite size is on the order of $10 \mathrm{~s}$ of $\mathrm{nm}$ and that there are twists of the orientation, perhaps in between the layers [4].

\section{References:}

[1] C Gammer et al, Ultramicroscopy 155 (2015), p. 1.

[2] CF Buitrago et al, Macromolecules 46 (2013), p. 9003.

[3] O. Panova et al, to be submitted.

[4] Work at the Molecular Foundry was supported by the Office of Science, Office of Basic Energy Sciences, of the U.S. Department of Energy under Contract No. DE-AC02-05CH11231. OP, XCC, NPB and AMM acknowledge the Electron Microscopy of Soft Matter Program from the Office of Science, Office of Basic Energy Sciences, Materials Sciences and Engineering Division of the U. S. Department of Energy under Contract No. DE-AC02-05CH11231. KIW, EBT and LY acknowledge support from the Miller Institute at the University of California Berkeley, NSF DMR (1506726) and ARO (W911NF-13-1-0363).

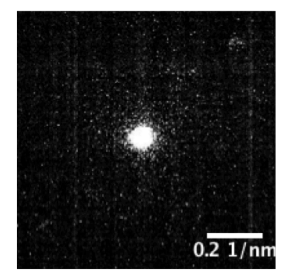

a.

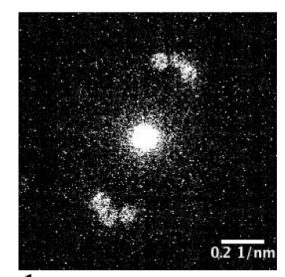

b.

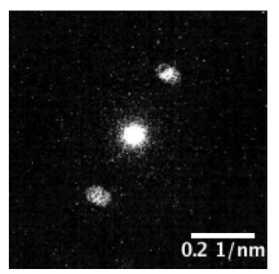

c.

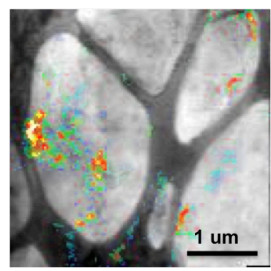

d.

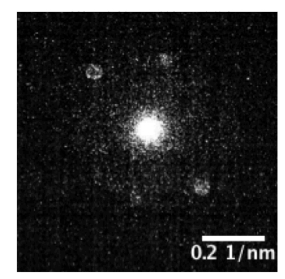

e.

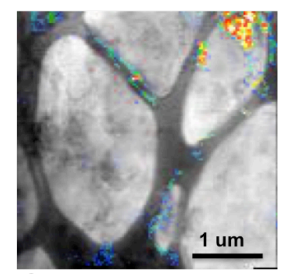

f.

Figure 1. Results from 4D data set of polyethylene. a) No diffraction condition; b) Diffraction from multiple crystallites either representing a twist during stacking or a probe sampling adjacent crystallites; c) Diffraction pattern from one orientation and d) real space reconstructed image showing where this diffraction orientation can be found; e) Diffraction pattern from a second orientation and f) real space reconstructed image. Images were acquired at $300 \mathrm{kV}$ using a $0.93 \mathrm{mrad}$ convergent probe with a probe size of $3-5 \mathrm{~nm}$. The STEM image is $128 \times 128$ pixels with exposure time of $2.5 \mathrm{~ms}$ per diffraction pattern. 